\title{
Multidimensional analysis of the learning curve for laparoscopic colorectal surgery in a regional hospital: the implementation of a standardized surgical procedure counterbalances the lack of experience
}

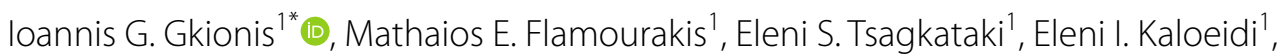
Konstantinos G. Spiridakis', Georgios E. Kostakis ${ }^{1}$, Athanasios K. Alegkakis ${ }^{2}$ and Manousos S. Christodoulakis ${ }^{1}$

\begin{abstract}
Background: Although a larger proportion of colorectal surgeries have been performed laparoscopically in the last few years, a steep learning curve prevents us from considering laparoscopic colorectal surgery as the gold standard technique for treating disease entities in the colon and rectum. The purpose of this single centre study was to determine, using various parameters and following a well-structured and standardized surgical procedure, the adequate number of cases after which a single surgeon qualified in open surgery but with no previous experience in laparoscopic colorectal surgery and without supervision, can acquire proficiency in this technique.

Methods: From 2012 to 2019, 112 patients with pathology in the rectum and colon underwent laparoscopic colorectal resection by a team led by the same surgeon. The patients were divided into two groups (group A:50 - group B:62) and their case records and histopathology reports were examined for predefined parameters, statistically analysed and compared between groups.

Results: There was no significant difference between groups in the distribution of conversions $(p=0.635)$ and complications ( $p=0.637$ ). Patients in both groups underwent surgery for the same median number of lymph nodes $(p=0.145)$ and stayed the same number of days in the hospital $(p=0.109)$. A statistically important difference was found in operation duration both for the total $(p=0.006)$ and for each different type of colectomy (sigmoidectomy: $p=0.026$, right colectomy: $p=0.013$, extralevator abdominoperineal resection: $p=0.050$, low anterior resection: $p=0.083$.
\end{abstract}

Conclusions: Taking into consideration all the parameters, it is our belief that a surgeon acquires proficiency in laparoscopic colorectal surgery after performing at least 50 diverse cases with a well structured and standardized surgical procedure.

Keywords: Learning curve, Colorectal, Laparoscopy, Standardized surgical procedure, Without supervision

*Correspondence: ioannisgionis@hotmail.com

1 Department of General Surgery, Venizeleio General Hospital, Leoforos

Knossou 44, Heraklion, Crete, Greece

Full list of author information is available at the end of the article

\section{Background}

Although the feasibility and oncologic efficacy of laparoscopic surgery for the treatment of inflammatory, benign and malignant disease entities in the colon and rectum 
have been demonstrated in randomized controlled trials [1-4], many surgeons are sceptical and avoid performing colorectal surgeries laparoscopically [5]. Four large prospective, randomized controlled trials, from North America, Canada and Europe, have been completed and have demonstrated that laparoscopic treatment of colon cancer yields oncologic results similar to those of open surgery, with no increased morbidity or mortality, and offers patients all the advantages of laparoscopic surgery [6-9]. Despite the fact that a greater adoption of laparoscopic colorectal surgery has been observed in recent years [10], the implementation of this technique is still progressing slowly [11]. According to recent statistical data, in England equal numbers of patients with pathology in the colon and rectum undergo open and laparoscopic surgery [12]. In other countries, the percentage of colorectal surgeries performed laparoscopically is much lower $[13,14]$. The main reason for this lower percentage is the steep and long-term learning curve of laparoscopic colorectal surgery $[15,16]$.

The term "learning curve", which was first introduced by Hermann Ebbinghaus in 1885 in the study of Psychology of Learning and Theodore Paul Wright in 1936 for the aircraft industry to express the graphic representation of the mean rate of learning for a procedure, has been imported into laparoscopic colorectal surgery and is under investigation by several studies [17-21]. A learning curve is completed when the predefined variables reach a steady state and the outcomes are comparable with those in the literature $[22,23]$.

Although multiple parameters and numerous criteria have been taken into consideration to determine the adequate number of cases to achieve proficiency, a consensus has not yet been reached among surgeons [24-26]. No reliable framework for case selection during training is available, and consequently, the learning curve for laparoscopic colorectal surgery has not been conclusively analysed [27].

The aim of this study was to determine the safety and clinical outcomes of laparoscopic resection for colorectal disease entities performed by a single surgeon with no previous experience in laparoscopic colorectal surgery and to analyse the learning curve of a well-structured and standardized surgical procedure followed by a standardized postoperative protocol [28] using various parameters.

\section{Methods}

From October 2012 to January 2019, 112 patients with pathology in the rectum and colon underwent laparoscopic colorectal resection at a regional general hospital (Venizeleio General Hospital of Heraklion in Crete), performed by a team led by the same surgeon (M.C.), who qualified for open surgery ( $>300$ colectomies) but had no previous experience with advanced laparoscopic procedures. Furthermore, laparoscopic colorectal operations were performed without the attendance or supervision of an experienced laparoscopic colectomy surgeon. Before beginning to perform colorectal resections laparoscopically, the surgeon (M.C.) became familiar with the cognitive aspects of this new procedure by watching operative videos, attending seminars and assisting during laparoscopic colorectal surgeries at specialized hospitals in this field. He obtained the necessary advanced laparoscopic surgery skills by training in animal models and virtual reality simulators.

Patients with locally advanced disease (T4 or bulky tumours), previous operations with a midline incision and BMI $>35$ were excluded from the study. The study population was organized chronologically according to the date of surgery and divided into two groups. The first group contained the initial 50 interventions, and the second group contained the following 62 patients. The case records and histopathology reports were examined for predefined parameters such as patient demographic data, location of the tumour, type of surgical procedure, conversion to open surgery, surgical time, distal and circumferential margins, number of harvested lymph nodes and total hospital stay. Mortality, surgical complications and oncological outcomes were also examined. The data collected (Additional file 1) were then statistically analysed and compared between these two groups.

The surgical time was calculated from the time of the first port placement to the time of wound closure, and the data for all laparoscopic surgeries were collected from the formal surgery records. Operations that were converted to open surgery were excluded from the surgical time analysis. The distal and circumferential margins and the number of harvested lymph nodes were obtained from the histopathology reports. Circumferential resection margins (CRMs) were defined as positive if malignant cells were found by microscopy at a distance of less than $2 \mathrm{~mm}$.

\section{Preoperative preparation}

All patients included in the present study underwent a preoperative preparation involving the following: extended medical report, physical examination, rectal exam, tests for the blood level of cancer markers, chest $\mathrm{X}$-ray, thoracic and abdominal computerized tomography and total colonoscopy with biopsy. Patients with a tumour in the rectum underwent rigid rectoscopy and nuclear magnetic resonance imaging (MRI) of the rectum. If preoperative chemoradiotherapy was conducted for the patients with a rectal tumour, a repetitive MRI was performed. 


\section{Surgical notes}

All surgical procedures were performed by a team led by the same surgeon (M.C.) without the supervision of any surgeon specialized in laparoscopic colorectal surgery. The rest of the team consisted of two trainees, (one responsible for the camera and the other as a surgical assistant), and a single scrub nurse. The role of each member of the team was precisely defined to make everything work correctly.

General anaesthesia with epidural analgesia was the preferred mode of anaesthesia. A well-structured and standardized surgical procedure was implemented following oncological criteria, according to the principle of complete mesocolic excision (CME) and total mesorectal excision (TME) with central vascular ligation (CVL) [29-31]. For patients with a tumour in the lower rectum that was unsuitable for low anterior resection, the chosen surgical technique was extralevator abdominoperineal resection (ELAPE) with the patient in a jackknife position [32, 33].

\section{Postoperative protocol}

All the patients in this study underwent a "fast track" postoperative protocol. Specifically, mobilization of the patients took place the next morning after the day of surgery. None of the patients had a nasogastric tube, so they were encouraged to drink liquids from the first postoperative day. The urinary catheter was removed the morning after the operation, with the exception of patients who underwent a low rectal resection or extralevator abdominoperineal resection. In these cases, the catheter was maintained for at least four days.

\section{Statistical methods}

The mean, median and standard deviation (SD) were used to describe the continuous variables, such as the number of operation hours and days of hospitalization, while frequencies and \%frequencies were used for discrete data. Independent samples t-test and the corresponding nonparametric Mann-Whitney test were used for two group comparisons. For comparisons of more than two groups, one-way ANOVA followed by Tukey's honest significant difference (HSD) post hoc test was used. Pearson's chi-square test was assessed to examine possible associations between two discrete variables. Box plots and scatter plots were used for graphical representation of the data. IBM SPSS Statistics 24.0 was used for statistical analysis of the data and an $a=0.05$ limit was set for accepting the null hypotheses.

\section{Results}

From October 2012 to January 2019, a total of 112 patients underwent laparoscopic colectomies at Venizeleio General Hospital of Heraklion in Crete (Greece). Patients were formally divided into two groups named A and B. Group A was characterised as the training group and group B was characterised as the post-training group. In Table 1, the demographics of the patients (age and sex) are shown. There was no significant difference in the patients' sex between groups A and B $(\mathrm{p}=0.546)$ or their age distribution $(\mathrm{p}=0.634)$.

Of a total of 112 patients who were initially selected for training and post-training laparoscopic surgery, nine (8.0\%) were excluded due to conversion from laparoscopic to open surgery (Table 2).

\section{Patient diagnoses and operation types}

The distribution of operation types in groups A and B is shown in Fig. 1. There was a higher number of sigmoidectomies in group $B(n=22)$ than in group A $(n=12)$, while the opposite pattern was shown for ELAPE operations, that is, $n=3$ for group $B$ vs $n=10$ for group A (Fig. 1). A significant difference in the frequencies of operation types was found for group A and B patients $(p=0.045)$.

\section{Number of lymph nodes, days of hospitalization, duration of surgery and clinical characteristics between the pre- and post-training periods}

In Table 3, the distribution of surgical margins and complications during the operation in the training (A) and post-training (B) groups are shown. Only 1 patient had positive margins, and this was recorded in group $A$. There was no significant difference in the type of margin proportions between the groups $(\mathrm{p}=0.301)$. Operational complications were observed in 7 patients $(6.8 \%)$.

Table 1 Sex and age distribution of patients between the training $(A)$ and post-training group (B)

\begin{tabular}{|c|c|c|c|c|c|}
\hline & \multicolumn{4}{|c|}{ Group } & \multirow[t]{3}{*}{$p$} \\
\hline & \multicolumn{2}{|l|}{$\mathbf{A}$} & \multicolumn{2}{|l|}{ B } & \\
\hline & $\mathbf{n}$ & $\%$ & $\mathbf{n}$ & $\%$ & \\
\hline \multicolumn{6}{|l|}{ Sex } \\
\hline $1(n=48)$ & 23 & 47.9 & 25 & 52.1 & 0.546 \\
\hline $2(n=64)$ & 27 & 42.2 & 37 & 57.8 & \\
\hline \multicolumn{6}{|l|}{ Age (10-years) } \\
\hline$\leq 50(n=13)$ & 5 & 38.5 & 8 & 61.5 & 0.634 \\
\hline $51-60(n=20)$ & 8 & 40.0 & 12 & 60.0 & \\
\hline $61-70(n=30)$ & 11 & 36.7 & 19 & 63.3 & \\
\hline $71-80(n=36)$ & 19 & 52.8 & 17 & 47.2 & \\
\hline $81+(n=13)$ & 7 & 53.8 & 6 & 46.2 & \\
\hline
\end{tabular}


Table 2 Distribution of operation conversion between the training group (A) and post-training group (B)

\begin{tabular}{|c|c|c|c|c|c|c|c|}
\hline & \multicolumn{4}{|c|}{ Group } & \multirow{2}{*}{\multicolumn{2}{|c|}{ Total }} & \multirow[b]{3}{*}{$p$} \\
\hline & \multicolumn{2}{|l|}{$\mathbf{A}$} & \multicolumn{2}{|l|}{ B } & & & \\
\hline & $\mathrm{n}$ & $\%$ & $\mathrm{n}$ & $\%$ & $\mathbf{n}$ & $\%$ & \\
\hline \multicolumn{8}{|c|}{ Conversion } \\
\hline No & 46 & 92.0 & 57 & 91.9 & 103 & 92.0 & 0.99 \\
\hline Yes & 4 & 8.0 & 5 & 8.1 & 9 & 8.0 & $(0.635)$ \\
\hline
\end{tabular}

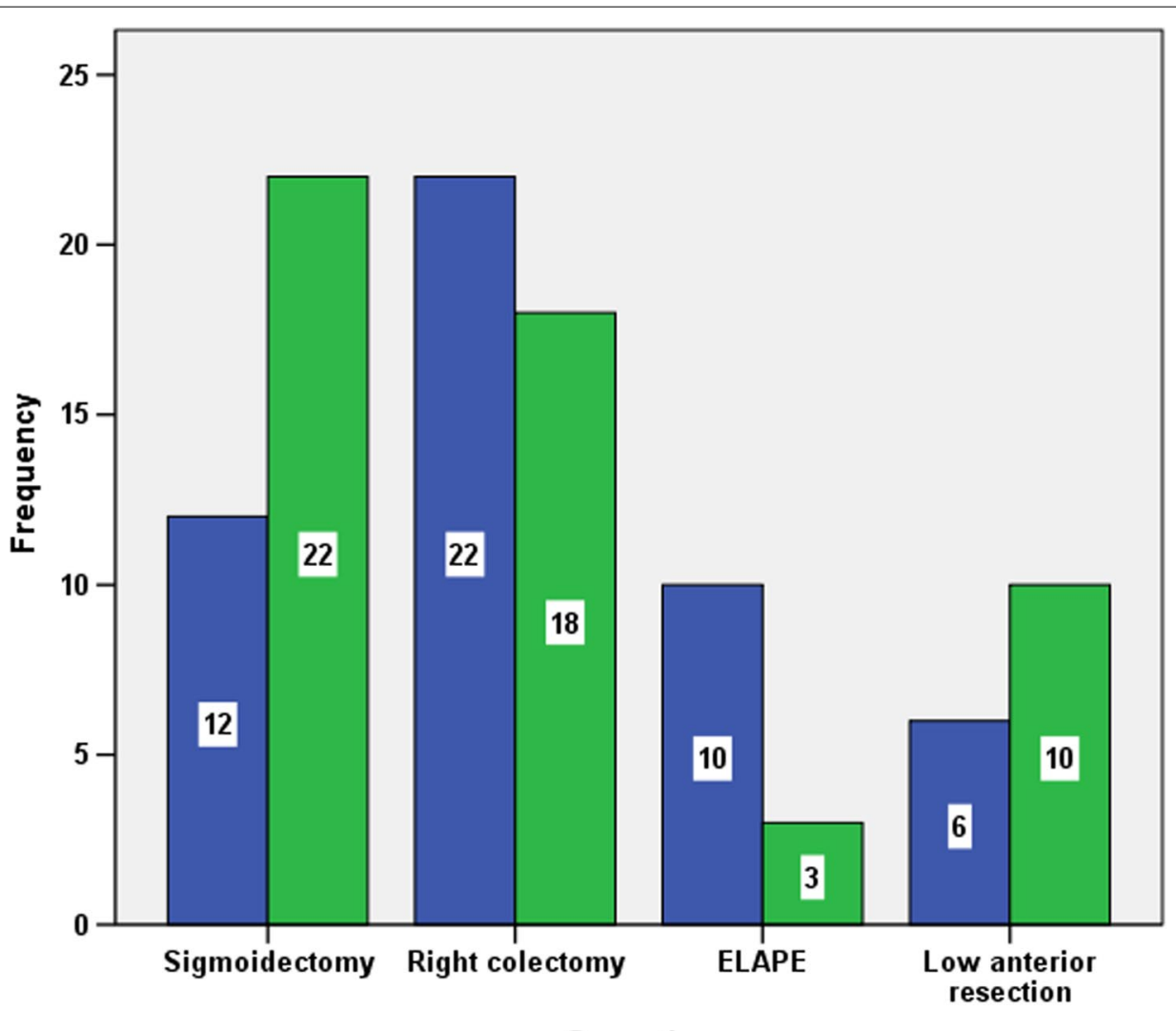

Group

$\square$ A

$\square$ B

Operation

Fig. 1 Distribution of operation types in groups A and B

Table 3 Distribution of complications during the operation and type of surgical margins

\begin{tabular}{|c|c|c|c|c|}
\hline \multicolumn{4}{|c|}{ Group } & \multirow[b]{3}{*}{$\mathbf{p}$} \\
\hline \multicolumn{2}{|l|}{ A } & \multicolumn{2}{|l|}{ B } & \\
\hline$n$ & $\mathrm{n}$ & $\mathrm{n}$ & $\%$ & \\
\hline 49 & 98.0 & 53 & 100.0 & 0.301 \\
\hline 1 & 2.0 & 0 & 0.0 & \\
\hline 46 & 92.0 & 50 & 94.3 & 0.637 \\
\hline 4 & 8.0 & 3 & 5.7 & \\
\hline
\end{tabular}

The distribution of complications was 4 cases in group $\mathrm{A}$ (8.0\%) and 3 cases in group B (5.7\%), which was not significantly different between groups $(\mathrm{p}=0.637)$.

In Tables 4 and 5, the complications between training (A) and post-training (B) groups and for each type of surgery, regardless group, are depicted. For each of these 7 patients with complications, a laparotomy was performed after the initial laparoscopic colorectal operation. In addition, the patient in group B who exhibited anastomotic leakage after sigmoidectomy died during the postoperative period of the second surgery due to deterioration of the respiratory system. No other 
Table 4 Description of complications between the two groups

\begin{tabular}{lll}
\hline Group & A & B \\
\hline & Complications & \\
Anastomotic leakage & 2 & 1 \\
Anastomotic stenosis & 1 & 0 \\
Ligature of the ureter & 1 & 0 \\
Lesion of the urinary bladder & 0 & 1 \\
Lesion of the iliac vein & 0 & 1 \\
Total number & 4 & 3 \\
\hline
\end{tabular}

deaths were reported during the laparoscopic colorectal surgeries or the immediate postoperative period.

Patients in both groups were operated on for the same median number of lymph nodes. The median number with quartile range of the lymph nodes for group A was 20 (14-28), while for group B, it was 26 (16-34), showing no statistically significant differences $(\mathrm{p}=0.145)$. Hospitalization days were not significantly different between the groups $(\mathrm{p}=0.109)$, showing a median of 7 (5-8) for group A and 6 (5-7) for group B. The operation duration was shorter in the posttraining group (group B) (median 4.0, 3.5-4.0 h) than in the training group (group A) (median 4.5, 3.5-5.5 h) $(\mathrm{p}=0.006)$ (Table 6).

\section{Surgical characteristics between the training group (A)} and post-training group (B) per type of operation

When the type of operation was considered during the A vs B group comparison, although the median surgical duration was lower in group B than in group A, significance was present only for low anterior resection 4.5 (4.0-4.5) hours for group B vs 5.5 (4.5-5.5) hours for group A $(p=0.036)$. A tendency for significance was found for sigmoidectomy $(p=0.059)$. Group B showed a median of $3.5(3.5-4.0)$ hours vs $4.5(3.5-5.0)$ hours for group A (Table 7).

\section{Timeline of the operation duration}

An alternative approach is shown in Fig. 2. The timeline of the operation duration in hours is presented vs consecutive patients' number for each of the operation types. Independent of the group (A or B), there was a significant decrease in operation time for sigmoidectomies $(\mathrm{rs}=-0.382, \mathrm{p}=0.026)$ as shown by the operation duration vs the patients' consecutive series. The same pattern of decline was found for right colectomy operations $(\mathrm{rs}=-0.389, \mathrm{p}=0.013)$ and ELAPE $(\mathrm{rs}=-0.554$, $\mathrm{p}=0.050$ ). A tendency towards decline was found for low anterior resection $(\mathrm{rs}=-0.446, \mathrm{p}=0.083)$.

Table 5 Description of complications for each type of surgery

\begin{tabular}{llll}
\hline Complications & \multicolumn{2}{l}{ Type of Surgery } & Low anterior resection \\
\cline { 2 - 4 } & Right colectomy & Sigmoidectomy & 2 \\
\hline Anastomotic leakage & - & 1 & - \\
Anastomotic stenosis & - & 1 & - \\
Ligature of the ureter & 1 & - & - \\
Lesion of the urinary bladder & - & 1 & - \\
Lesion of the iliac vein & - & 1 & 2 \\
Total number & 1 & 4 & -
\end{tabular}

Table 6 Number of lymph nodes, days of hospitalization and operation duration between the training (group A) and post-training group (group B)

\begin{tabular}{|c|c|c|c|c|c|c|c|c|c|c|c|}
\hline & \multicolumn{10}{|l|}{ Group } & \multirow{4}{*}{$\mathbf{p}^{*}$} \\
\hline & \multicolumn{5}{|l|}{$A$} & \multicolumn{5}{|l|}{ B } & \\
\hline & \multirow[t]{2}{*}{ Mean } & \multirow[t]{2}{*}{ SD } & \multicolumn{3}{|c|}{ Quartiles } & \multirow[t]{2}{*}{ Mean } & \multirow[t]{2}{*}{ SD } & \multicolumn{3}{|c|}{ Quartiles } & \\
\hline & & & $1 s t$ & 2nd & $3 r d$ & & & $1 s t$ & 2nd & $3 r d$ & \\
\hline Operation duration (hours) & 4.5 & 1.2 & 3.5 & 4.5 & 5.5 & 4.0 & 1.4 & 3.5 & 4.0 & 4.0 & 0.006 \\
\hline Hospitalization (days) & 7.9 & 4.7 & 5.0 & 7.0 & 8.0 & 6.2 & 1.9 & 5.0 & 6.0 & 7.0 & 0.109 \\
\hline Lymph nodes (number) & 22.3 & 12.2 & 14.0 & 19.5 & 28.0 & 26.8 & 15.1 & 16.0 & 26.0 & 34.0 & 0.145 \\
\hline
\end{tabular}

* Mann-Whitney test 
Table 7 Differences in surgical characteristics between the training group (A) and post-training group (B) per type of operation

\begin{tabular}{|c|c|c|c|c|c|c|c|c|}
\hline & \multirow[b]{4}{*}{ Operation } & \multicolumn{6}{|c|}{ Group } & \multirow[b]{4}{*}{$\mathbf{P}$} \\
\hline & & \multicolumn{3}{|l|}{ A } & \multicolumn{3}{|l|}{ B } & \\
\hline & & \multicolumn{3}{|c|}{ Quartile } & \multicolumn{3}{|c|}{ Quartile } & \\
\hline & & $1 \mathrm{st}$ & 2nd & $3 r d$ & $1 \mathrm{st}$ & 2nd & $3 r d$ & \\
\hline \multirow[t]{4}{*}{ Duration (hours) } & Sigmoidectomy & 3.5 & 4.5 & 5.0 & 3.5 & 3.5 & 4.0 & 0.059 \\
\hline & Right colectomy & 3.5 & 4.0 & 5.0 & 3.5 & 3.5 & 4.0 & 0.286 \\
\hline & ELAPE & 4.5 & 6.0 & 6.0 & 4.3 & 5.0 & 5.8 & 0.937 \\
\hline & Low anterior resection & 4.5 & 5.5 & 5.5 & 4.0 & 4.5 & 4.5 & 0.036 \\
\hline \multirow[t]{4}{*}{ Hospitalization (days) } & Sigmoidectomy & 6 & 6 & 8 & 5 & 5 & 7 & 0.557 \\
\hline & Right colectomy & 5 & 7 & 8 & 5 & 6 & 7 & 0.697 \\
\hline & ELAPE & 7 & 8 & 9 & 7 & 9 & 10 & 0.371 \\
\hline & Low anterior resection & 6 & 7 & 8 & 6 & 6 & 7 & 0.713 \\
\hline \multirow[t]{4}{*}{ Lymph nodes (number) } & Sigmoidectomy & 14 & 17 & 27 & 12 & 23 & 33 & 0.901 \\
\hline & Right colectomy & 18 & 22 & 28 & 20 & 28 & 36 & 0.240 \\
\hline & ELAPE & 7 & 14 & 16 & 16 & 18 & 37 & 0.077 \\
\hline & Low anterior resection & 15 & 25 & 29 & 20 & 28 & 34 & 0.713 \\
\hline
\end{tabular}

\section{Discussion}

Laparoscopic colorectal surgery is demanding because it requires an elevated level of technical skills. Although the feasibility and oncologic efficacy of laparoscopic colorectal surgery have been proven [1-4], its implementation in daily surgical practice is still limited $[11,13,14]$. The main factor contributing to this limitation is the steep and long-term learning curve $[15,16]$. In the literature, the number of cases needed to achieve proficiency in laparoscopic colorectal surgery varies enormously. Simons et al. reported a learning curve of 11-15 cases in a series of 144 patients in 1995 [34], whereas Tekkis et al. demonstrated a learning curve of 55 cases for right-sided colectomies versus 62 cases for left-sided resections [17]. In a multicentre analysis of 4852 cases, the learning curves varied from 87 to 152 procedures [27]. In other studies $[15,25,26,35,36]$, the adequate number of laparoscopic resections ranged from 30 to 70 cases. As can easily be understood, there is no consensus among surgeons [2426]. Therefore, the aim of this study was to evaluate the learning curve based on the initial outcomes of our first 112 operations using various parameters (Additional file 1).

The present study demonstrates that conversion rates do not differ significantly as the surgeon gains more experience. Our overall $8 \%$ conversion rate is in accordance with the $5-20 \%$ reported in the literature [37-39] and there was no significant change between the first 50 and the next 62 cases. Furthermore, no significant difference in the rate of surgical complications was identified between the two groups. Operational complications were observed in 7 cases among the total number of patients (6.8\%), and the distribution was 4 cases in group A (8.0\%) and 3 cases in group B (5.7\%). Other reported series have concluded that at least $40-50$ procedures are necessary to significantly lower the complication rate [20, 26, 34]. In addition, the $0.8 \%$ overall mortality rate and the $2.9 \%$ anastomotic leak rate for laparoscopic surgeries (3 cases of low anterior resection), which were detected in our study, are comparable with those in multicentre trials [37-39].

A very important parameter is the oncologic efficacy of the laparoscopic colorectal procedure. That goal, as represented by negative surgical distal and circumferential margins and an adequate number of harvested lymph nodes, can be reached early in the learning curve, as demonstrated in our research. The median and quartile range of lymph nodes for the training and post-training groups were comparable, showing no statistically significant difference. Furthermore, only one patient in the training group with a bulky tumour in the rectum locally expanded, despite neoadjunant chemotherapy and radiotherapy, had positive circumferential surgical margins. These results can be easily explained by taking into consideration the ample knowledge of anatomy and the respect for the rules of surgical oncology, which are credentials of the surgeon that are obtained by experience in open surgery. Inappropriate resection is not justifiable even in the training period, and oncological outcomes should not be compromised. For this reason, the completion of the operation laparoscopically does not constitute a purpose in itself. 


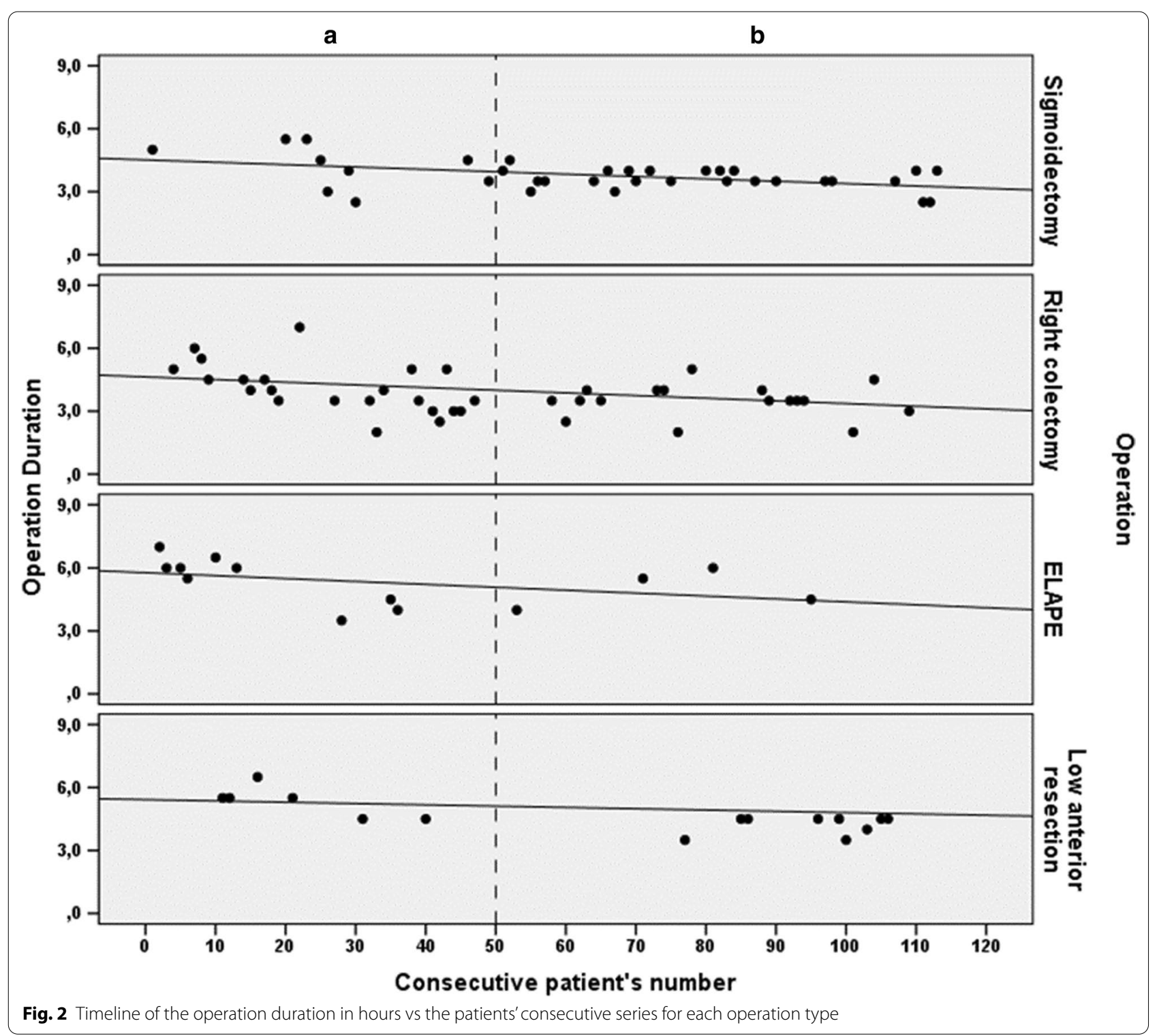

The increasing number of cases performed laparoscopically did not alter the overall hospitalization of the patients between the two groups. The implementation of a well-structured and standardized surgical procedure followed by a standardized postoperative protocol ensures that all patients take advantage of the benefits of laparoscopic surgery [6-9]. COLOR II and Ivanov P et al. reported reduced hospitalization as a result of faster recovery in laparoscopically operated patients versus those who had an open surgery $[40,41]$.

The operation time in laparoscopic colorectal surgery is longer than that in open surgery even if the resections are performed by surgeons with experience in advanced laparoscopic procedures [42]. In our experience, the operating time decreases with the surgeon's increasing experience, and it is a useful criterion for evaluating the learning curve. Specifically, there was a significant decrease in operation time for sigmoidectomies, right colectomy operations and ELAPE, and a tendency towards decline was found for low anterior resections. Our results are similar to those of Choi et al., who demonstrated a decline in the duration of laparoscopic sigmoidectomy after 30-42 cases [35].

\section{Conclusions}

The key factor for accomplishing adequate oncologic resections laparoscopically and for shortening the learning curve is the implementation of a 
well-structured and standardized surgical technique followed by a standardized postoperative protocol. From our perspective, a surgeon acquires proficiency in laparoscopic colorectal surgery after performing at least 50 diverse cases. It is our belief that the results of this study will encourage and enable a larger number of surgeons to adopt laparoscopic surgery in daily clinical practice as the gold standard technique for treating diseases in the colon and rectum, even if they do not have previous experience or they work in a regional hospital.

\section{Supplementary information}

Supplementary information accompanies this paper at https://doi. org/10.1186/s12893-020-00975-6.

Additional file 1. Data set created by the examination of the case records and histopathology reports of the 112 patients who underwent laparoscopic colorectal interventions.

\section{Abbreviations}

M.C.: Manousos Christodoulakis; CRM: Circumferential resection margins; MRI: Magnetic resonance imaging; CME: Complete mesocolic excision; TME: Total mesorectal excision; CVL: Central vascular ligation; ELAPE: Extralevator abdominoperineal resection; SD: Standard deviation; HSD: Honest significant difference.

\section{Acknowledgements}

Not applicable.

\section{Authors' contributions}

All authors contributed to the study conception and design. Data collection and analysis were performed by IG, MF, ET, EK and AA. MC supervised the project. The first draft of the manuscript was written by IG in consultation with KS, GK and AA. All authors commented on previous versions of the manuscript. All authors read and approved the final manuscript.

\section{Funding}

There was no funding.

\section{Availability of data and materials}

The datasets generated and analysed during the current study are included in this manuscript and its supplementary information files.

\section{Ethics approval and consent to participate}

This study was conducted in accordance with the ethical standards of the institutional and national research committee and with the 1964 Helsinki Declaration and its later amendments or comparable ethical standards. The study was approved by the Ethics Committee of the Scientific Board of Venizeleio General Hospital of Heraklion (Decision No. 128/Meeting 17/18-12-2019). Informed consent was obtained from all patients. The consent was either written or verbal. From some patients, verbal consent was obtained due to either a low level of educational attainment or a better understanding of the scientific protocol of the study using oral language. In these cases, a member of the Ethics Committee of the Scientific Board of Venizeleio General Hospital of Heraklion was always present, in order to ensure the understanding of the scientific protocol of the study from the patients and to certify that they give their consent. The Ethics Committee of the Scientific Board of Venizeleio General Hospital of Heraklion, with the same decision (Decision No.128/Meeting 17/18-12-2019), accepted both methods (written or verbal) for providing information to and obtaining consent from the patients.

\section{Consent for publication}

Not applicable.

\section{Competing interests}

The authors declare no conflicts of interest.

\section{Author details}

${ }^{1}$ Department of General Surgery, Venizeleio General Hospital, Leoforos Knossou 44, Heraklion, Crete, Greece. ${ }^{2}$ Medical School, University of Crete, Andrea Kalokerinou 13, Heraklion, Crete, Greece.

Received: 14 Auqust 2020 Accepted: 19 November 2020

Published online: 02 December 2020

\section{References}

1. Lacy AM, Garcia-Valdecasas JC, Delgado S, et al. Laparoscopy assisted colectomy versus open colectomy for treatment of non-metastatic colon cancer: a randomised trial. Lancet. 2002;359(9325):2224-9.

2. Clinical Outcomes of Surgical Therapy Study Group, Nelson H, Sargent DJ, Wieand HS, Fleshman J et al. (2004) A comparison of laparoscopically assisted and open colectomy for colon cancer. N Engl J Med 350(20):2050-9.

3. Veldkamp R, Kuhry E, Hop WC, et al. Colon cancer Laparoscopic or Open Resection Study Group (COLOR). Laparoscopic surgery versus open surgery for colon cancer: short-term outcomes of a randomised trial. Lancet Oncol. 2005;6:477-84

4. Braga M, Frasson M, Zuliani W, et al. Randomized clinical trial of laparoscopic versus open left colonic resection. Br J Surg. 2010;97:1180-6.

5. Schwab KE, Dowson HM, van Dellen J, et al. The uptake of laparoscopic colorectal surgery in Great Britain and Ireland: a questionnaire survey of consultant members of the ACPGBI. Colorectal Dis. 2009:11(3):318e22.

6. Lacy AM, Delgado S, Castells A, et al. The long-term results of a randomized clinical trial of laparoscopy-assisted versus open surgery for colon cancer. Ann Surg. 2008;248(1):1e7.

7. Fleshman J, Sargent DJ, Green E, et al. Laparoscopic colectomy for cancer is not inferior to open surgery based on 5-year data from the COST study group trial. Ann Surg. 2007;246(4):654e5.

8. Guillou PJ, Quirke P, Thorpe $H$, et al. Short-term endpoints of conventional versus laparoscopic-assisted surgery in patients with colorectal cancer (MRC CLASICC trial): multicentre, randomised controlled trial. Lancet. 2005;365(9472):1718e26.

9. Luglio G, Nelson H. Laparoscopy for colon cancer: state of the art. Surg Oncol Clin N Am. 2010;19(4):777e91.

10. Bardakcioglu O, Khan A, Aldridge C, et al. Growth of laparoscopic colectomy in the United States: analysis of regional and socioeconomic factors over time. Ann Surg. 2013;258(2):270e4.

11. Kemp JA, Finlayson SR. Nationwide trends in laparoscopic colectomy from 2000-2004. Surg Endosc. 2008;22:1181-7.

12. Laudicella $M$, Walsh $B$, Munasinghe $A$, et al. Impact of laparoscopic versus open surgery on hospital costs for colon cancer: a population-based retrospective cohort study. BMJ open. 2016;6:e012977.

13. Rea JD, Cone MM, Diggs BS, et al. Utilization of laparoscopic colectomy in the United States before and after the clinical outcomes of surgical therapy study group trial. Ann Surg. 2011;254(2):281-8.

14. Prakash K, Kamalesh NP, Pramil K, et al. Does case selection and outcome following laparoscopic colorectal resection change after initial learning curve? Analysis of 235 consecutive elective laparoscopic colorectal resections. J Minim Access Surg. 2013;9(3):99-103.

15. Schlachta CM, Mamazza J, Seshadri PA, et al. Defining a learning curve for laparoscopic colorectal resections. Dis Colon Rectum. 2001;44:217-22.

16. Luján J, Gonzalez A, Abrisqueta J, et al. The learning curve of laparoscopic treatment of rectal cancer does not increase morbidity. Cir Esp. 2014;92(7):485-90.

17. Tekkis PP, Senagore AJ, Delaney CP, et al. Evaluation of the learning curve in laparoscopic colorectal surgery: comparison of right-sided and leftsided resections. Ann Surg. 2005;242:83-91.

18. Park IJ, Choi GS, Lim KH, et al. Multidimensional analysis of the learning curve for laparoscopic colorectal surgery: lessons from 1,000 cases of laparoscopic colorectal surgery. Surg Endosc. 2009;23:839-46.

19. Wishner JD, Baker JW Jr, Hoffman GC, et al. Laparoscopic-assisted colectomy. The learning curve Surg Endosc. 1995;9:1179-83. 
20. Agachan F, Joo JS, Sher M, et al. Laparoscopic colorectal surgery. Do we get faster? Surg Endosc. 1997;11:331-5.

21. Khan N, Abboudi H, Khan MS, et al. Measuring the surgical "learning curve": methods, variables and competency. BJU Int. 2014;113(3):504-8.

22. Cuschieri A. Nature of human error: implications for surgical practice. Ann Surg. 2006;244:642-8.

23. Pitiakoudis M, Michailidis L, Zezos $\mathrm{P}$, et al. Quality training in laparoscopic colorectal surgery: does it improve clinical outcome? Tech Coloproctol. 2011;15(Suppl 1):S17-20.

24. Dincler S, Koller MT, Steurer J, et al. Multidimensional analysis of learning curves in laparoscopic sigmoid resection: eight-year results. Dis Colon Rectum. 2003:46:1371-8 ((discussion 1378-1379)).

25. Senagore AJ, Luchtefeld MA, Mackeigan JM. What is the learning curve for laparoscopic colectomy? Am Surg. 1995;61:681-5.

26. Bennett CL, Stryker SJ, Ferreira MR. The learning curve for laparoscopic colorectal surgery. Preliminary results from a prospective analysis of 1194 laparoscopic- assisted colectomies. Arch Surg. 1997;132:41-4.

27. Miskovic D, Ni M, Wyles SM, et al. Learning curve and case selection in laparoscopic colorectal surgery: systematic review and international multicenter analysis of 4852 cases. Dis Colon Rectum. 2012:55(12):1300-10.

28. Akiyoshi T, Kuroyanagi H, Ueno M, et al. Learning curve for standardized laparoscopic surgery for colorectal cancer under supervision: a singlecenter experience. Surg Endosc. 2011;25(5):1409-14.

29. West NP, Hohenberger W, Weber K, et al. Complete mesocolic excision with central vascular ligation produces an oncologically superior specimen compared with standard surgery for carcinoma of the colon. J Clin Oncol. 2010;28(2):272e8.

30. West NP, Sutton KM, Ingeholm P, et al. Improving the quality of colon cancer surgery through a surgical education program. Dis Colon Rectum. 2010;53(12):1594e603.

31. Luglio G, de Palma GD, Tarquini R, et al. (2015) Laparoscopic colorectal surgery in learning curve: Role of implementation of a standardized technique and recovery protocol. A cohort study. Ann Med Surg. 2015;4(2):89-94

32. Kamali D, Sharpe A, Musbahi A, et al. Oncological and quality of life outcomes following extralevator versus standard abdominoperineal excision for rectal cancer. Ann R Coll Surg Engl. 2017;99(5):402-9.
33. Zhang $Y$, Wang $D$, Zhu L, et al. Standard versus extralevator abdominoperineal excision and oncologic outcomes for patients with distal rectal cancer: a meta-analysis. Medicine (Baltimore). 2017;96(52):e9150.

34. Simons AJ, Anthone GJ, Ortega AE, et al. Laparoscopic assisted colectomy learning curve. Dis Colon Rectum. 1995;38:600-3.

35. Choi DH, Jeong WK, Lim SW, et al. Learning curves for laparoscopic sigmoidectomy used to manage curable sigmoid colon cancer: singleinstitute, three-surgeon experience. Surg Endosc. 2009;23:622-8.

36. Avital $\mathrm{S}$, Hermon $\mathrm{H}$, Greenberg $\mathrm{R}$, et al. Learning curve in laparoscopic colorectal surgery: our first 100 patients. Isr Med Assoc J. 2006;8(10):683-6.

37. Rose J, Schneider C, Yildirim C, et al. Complications in laparoscopic colorectal surgery: results of a multicentre trial. Tech Coloproctol. 2004;8(Suppl 1):s25-8.

38. De Mulder W, Gillardin JP, Hofman P, et al. Laparoscopic colorectal surgery. Analysis of the first 237 cases. Acta Chir Belg. 2001;101(1):25-30.

39. Köckerling F, Schneider C, Reymond MA, et al. Early results of a prospective multicenter study on 500 consecutive cases of laparoscopic colorectal surgery Laparoscopic Colorectal Surgery Study Group (LCSSG). Surg Endosc. 1998;12(1):37-41.

40. Van der Pas MH, Haglind E, Cuesta MA, et al. Laparoscopic versus open surgery for rectal cancer (COLOR II): short-term outcomes of a randomised, phase 3 trial. Lancet Oncol. 2013;14(3):210-8.

41. Ivanov P, Vasilev K, Kotashev G, et al. Laparoscopic vs open resection for rectal carcinoma-a prospective analysis. Khirurgiia (Sofiia). 2013;1:23-9.

42. Lezoche E, Feliciotti F, Paganini AM, et al. Laparoscopic colonic resections versus open surgery: a prospective non-randomized study on 310 unselected cases. Hepatogastroenterology. 2000;47(33):697-708.

\section{Publisher's Note}

Springer Nature remains neutral with regard to jurisdictional claims in published maps and institutional affiliations.
Ready to submit your research? Choose BMC and benefit from:

- fast, convenient online submission

- thorough peer review by experienced researchers in your field

- rapid publication on acceptance

- support for research data, including large and complex data types

- gold Open Access which fosters wider collaboration and increased citations

- maximum visibility for your research: over $100 \mathrm{M}$ website views per year

At BMC, research is always in progress.

Learn more biomedcentral.com/submissions 